\title{
Advanced Magnetic Resonance Imaging Findings of Multinodular and Vacuolating Neuronal Tumor
}

\author{
Aynur TURAN, Idil Gunes TATAR, Azad HEKIMOGLU, Huseyin COSKUN, Ferhat YILDIRIM \\ University of Health Sciences, Diskapi Yildirim Beyazit Health Application and Research Center, Radiology Department, Ankara, Turkey \\ This study has been presented as a poster at the $27^{\text {th }}$ Year Annual Meeting of Neuroradiology and Head-Neck Radiology, Turkish Society of \\ Neuroradiology between 16 and 18 February 2018 at Istanbul, Turkey.
}

Corresponding author: Aynur TURAN aturanrad@gmail.com

\section{ABSTRACT}

AIM: To present the magnetic resonance imaging (MRI) findings of a multinodular and vacuolating neuronal tumor (MVNT).

MATERIAL and METHODS: The authors identified four patients with MVNT in the hospital between January 2015 and October 2019. Both the clinical and radiological data of the patients were collected for analysis.

RESULTS: Three patients complained of non-specific headaches. One patient had vertigo and imbalance. MRI sequences, including spectroscopy, perfusion, and DWI sequences, were retrospectively evaluated. The lesions were located in the subcortical and periventricular white matter of the parietal and temporal lobes, showed confluency, and comprised nodular pattern. The lesions appeared isointense to the cerebral cortex on T1 weighted imaging and hyperintense on T2 weighted and FLAIR sequences. None of the lesions showed diffusion restriction or contrast enhancement. Three of the lesions demonstrated a slight increase in choline peak and a slight decrease in $\mathrm{N}$-acetyl aspartate peak. One lesion showed a noticeable increase in the Cho peak and a decrease in the NAA peak.

CONCLUSION: Radiological features of MVNT are specific. Recognizing the MRI findings would help avoid unnecessary interventions in these patients, who are usually asymptomatic.

KEYWORDS: Multinodular and vacuolating neuronal tumor, Magnetic resonance imaging, Advanced imaging

ABBREVIATIONS: MVNT: Multinodular and vacuolating neuronal tumor, MRI: Magnetic resonance imaging, DWI: Diffusionweighted imaging, RIS: Radiological Information System, Cho: Choline, NAA: N-acetyl aspartate, CBV: Cerebral blood volume, Cr: Creatine, WHO: World Health Organisation, CNS: Central Nervous System, DNET: Dysembryoplastic neuroepithelial tumors, FCD: Focal cortical dysplasia, PVS: Periventricular space

\section{INTRODUCTION}

$\mathrm{T}$ The multinodular and vacuolating neuronal tumor (MVNT) was first described pathologically as a benign neuronal lesion by Huse et al. in 2013, after working on a series of ten patients (8). MVNT is located in cerebral hemispheres, mainly in the temporal and parietal lobes, with a predilection for subcortical white matter and deep cortical layers (13). The middle age group is usually affected. Clinically, patients are generally asymptomatic, and the lesion is noticed incidentally in magnetic resonance imaging (MRI), although some may show seizures or seizure equivalents $(1,8,13)$. Since MVNT is a newly defined entity with a limited number of cases in the literature, awareness of this diagnosis is low among radiologists and clinicians. Therefore, to demonstrate the nature of this entity, advanced MRI sequences are usually employed. The literature reports insufficient and contradicting information about the advanced MRI findings of MVNT. With
Aynur TURAN (10: 0000-0001-6654-3129

Idil Gunes TATAR (1) : 0000-0003-2499-4532

Azad HEKIMOGLU (1) : 0000-0001-7853-019X
Huseyin COSKUN (10): 0000-0001-5405-3544

Ferhat YILDIRIM (D) : 0000-0002-4510-0406 
this case series, the authors intend to demonstrate and discuss the radiologic features of MVNT, including diffusionweighted imaging (DWI), MR spectroscopy, and MR perfusion imaging.

\section{- MATERIAL and METHODS}

The electronic information system of the hospital was retrospectively searched from January 2015 to October 2019. Four patients were identified with MVNT. Two experienced neuroradiologists retrospectively reanalyzed the conventional and advanced MRI examinations of the four patients. The location, size, shape, contour, signal intensity, contrast enhancement, diffusion characteristics, perfusion, and spectroscopic characteristics of the lesions were analyzed. The patients' demographic findings, such as age and sex, history, clinical and laboratory findings, were retrieved from the Radiological Information System (RIS).

\section{口 RESULTS}

One of the four patients was male, while the remaining three were females. The median age of the patients was 45 (40-52 years). One patient had vertigo, and the other three patients had headaches. One out of the four patients had a history of trauma dating back to five years ago, and one of them had hypertension. None of the patients presented focal neurologic symptoms. The whole blood count and biochemical findings of all the patients were normal. Since the patients did not undergo biopsy or operation, pathology results were not obtained. Typical MRI findings helped accomplish the patients' diagnosis, and no changes were observed in the 1-3 years radiological follow-up.

\section{MRI Findings}

All the patients underwent contrast-enhanced cranial MRI, DWI, and multivoxel spectroscopic examinations. Only one patient (Case 1) underwent an MR perfusion examination. The lesions' MRI findings were similar as they were all located in the cortical-juxtacortical white matter with cluster formation, consisting of multiple round or oval nodules. All the lesions were identified as iso-hypointense on $\mathrm{T} 1$ and hyperintense on T2 weighted and fluid-attenuated inversion recovery (FLAIR) sequence. The lesions did not show mass effect, edema, diffusion restriction, or contrast enhancement. Three of the lesions (Case 1,2,4) demonstrated a slight increase in choline (Cho) peak and a slight decrease in N-acetyl aspartate (NAA) peak. One of the lesions showed (Case 3 ) a notable increase in Cho peak and a reduction in NAA peak. The cerebral blood volume (CBV) measurements MR perfusion showed a slightly decreased perfusion than the rest of the brain in one of the lesions.

Case 1: The lesion was located in the right parietal lobe, extending from the subcortical to the periventricular white matter. It measured $38 \times 28 \mathrm{~mm}$, forming clusters of millimetric sized peripheral satellite nodules (Figure 1A). The MR perfusion CBV map of the lesion showed slightly decreased perfusion than the rest of the brain (Figure 1B). The MR spectroscopy revealed a slight increase in Cho peak (Cho/Cr was 1.33) and a slight decrease in NAA peak (Cho/NAA was 0.71) (Figure $1 \mathrm{C})$. No progression in the lesion was detected in the three years follow-up.

Case 2: The lesion was located in the left parietal lobe, within the cortical-subcortical white matter, measuring $27 \times 18 \mathrm{~mm}$ (Figure 2A, B). The deep layers of the surrounding cortex were

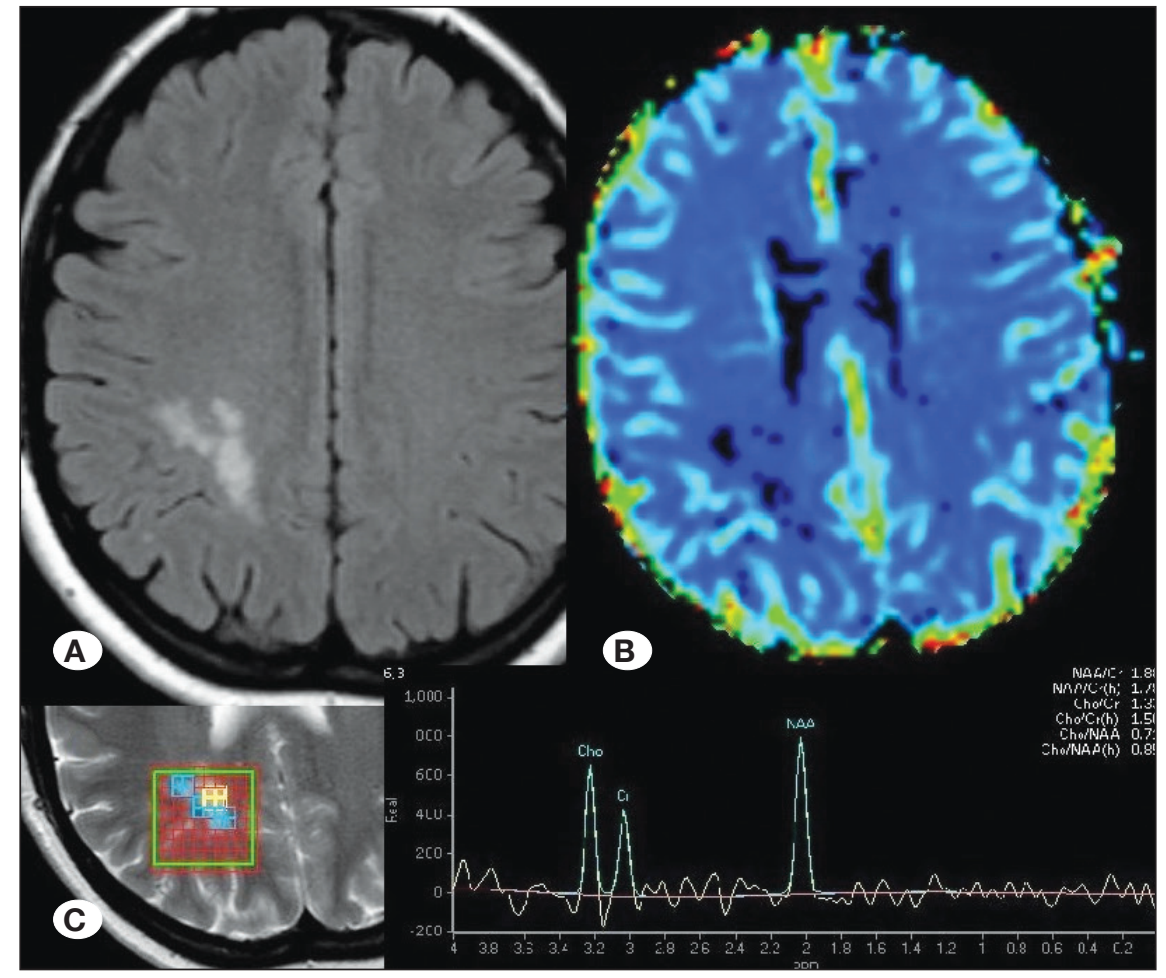

Figure 1: In the right parietal lobe, hyperintense clusters formed with millimetric sized peripheral satellite nodules can be seen in the FLAIR sequence (A). MR perfusion CBV map of the lesion shows slightly decreased perfusion than the rest of the brain (B). MR spectroscopy shows a slight increase in Cho peak and a slight decrease in NAA peak; Cho/Cr was measured to be 1.33, Cho/ NAA was detected to be 0.71 (C). 
affected and appeared thinner. The MR spectroscopy revealed an increase in Cho peak (Cho/Cr was 1.18) and a decrease in NAA peak (Cho/NAA was 1.01) (Figure 2C). In the five year follow-up, no change in the lesion was detected. The patient's diagnosis was made in the last two years, previously reported as a non-specific lesion with benign nature.

Case 3: The lesion was located in the right parahippocampal region, measuring $54 \times 28 \mathrm{~mm}$. The hippocampal cortex appeared thin, and there were multiple millimetric satellite nodules around the cortex (Figure 3A, B). Cho was prominently increased (Cho/Cr was 1.74), and NAA was decreased (Cho/ NAA was 2.00) (Figure 3C). The one-year follow-up did not show any change in the lesion.

Case 4: The lesion was located in the left parietal lobe of the subcortical white matter and measured $25 \times 26 \mathrm{~mm}$, forming clusters (Figure 4A, B). The MR spectroscopy revealed a minimal increase in Cho peak (Cho/Cr was 1.03) and a minimal decrease in NAA peak (Cho/NAA was 0.65) (Figure 4C). No progression in the lesion change was detected in the one-year follow-up.
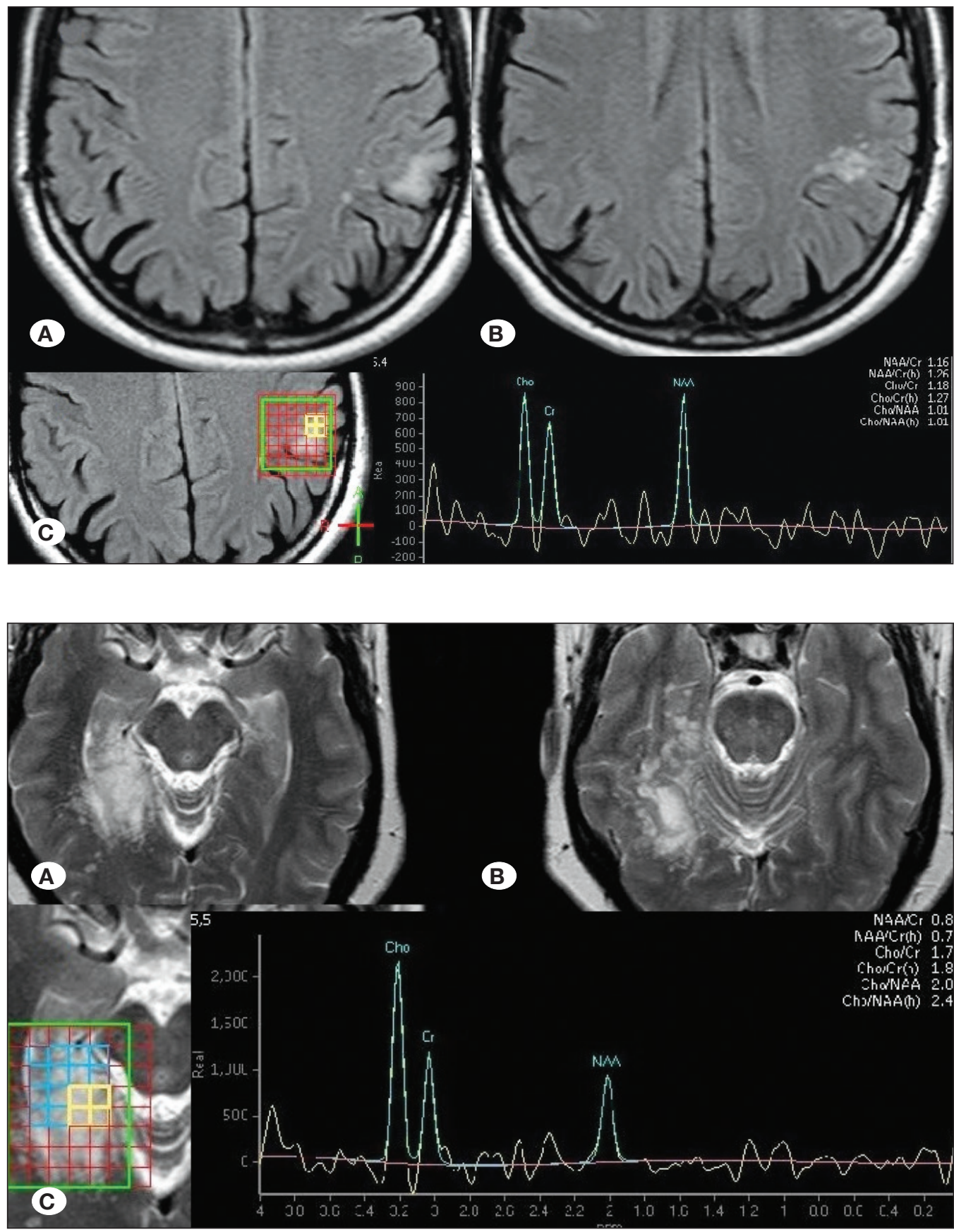

Figure 2: A hyperintense lesion located in the cortexsubcortical white matter of the left parietal lobe (A,B). The lesion's MR spectroscopy reveals an increase in Cho peak and a decrease in NAA peak; Cho/ Cr was 1.18, Cho/NAA was 1.01 (C).
Figure 3: MR spectroscopy of the parahippocampal lesion exhibited a prominently increased Cho and decreased NAA. Cho/Cr was 1.74, Cho/ NAA was 2.00 . 


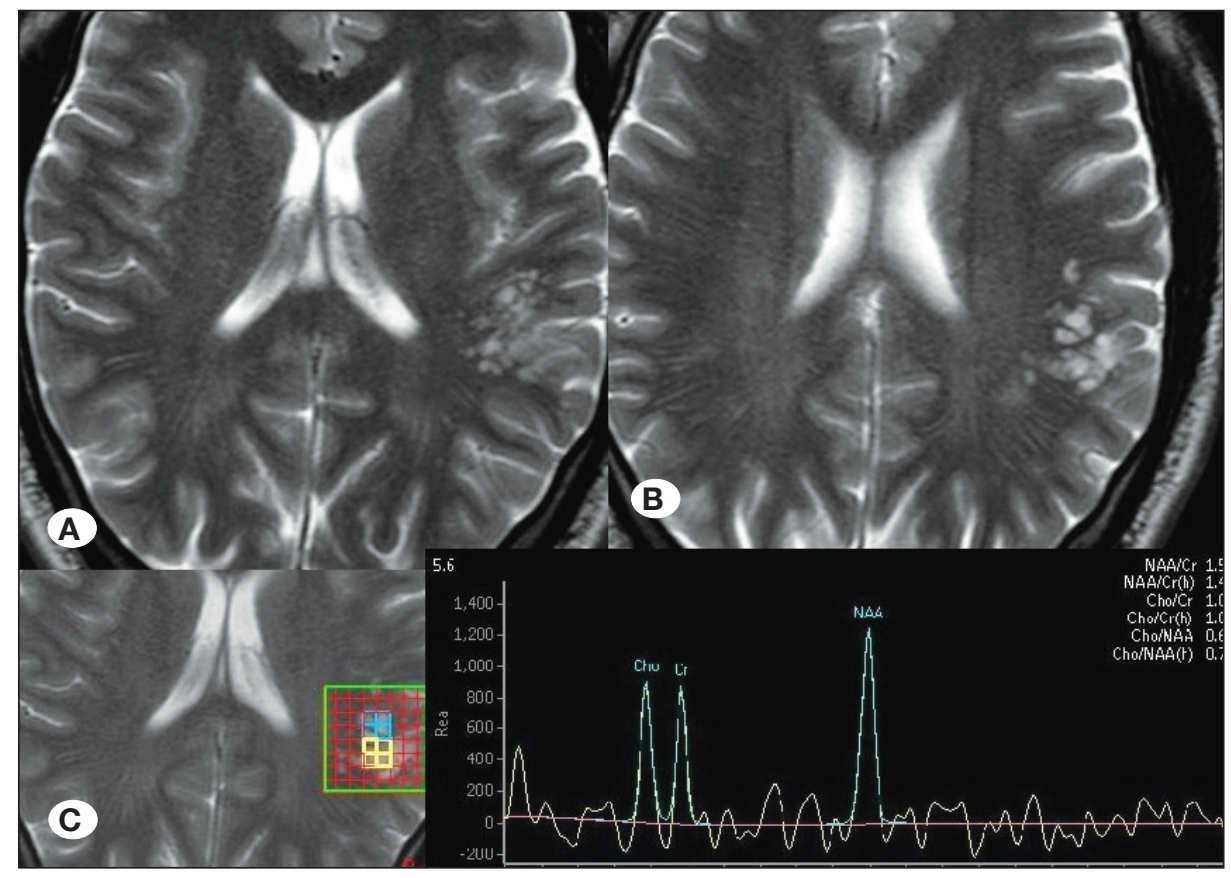

Figure 4: MR spectroscopy of subcortical white matter of the left parietal lobe shows a minimal increase in Cho peak and a minimal decrease in NAA peak; Cho/Cr was 1.03, Cho/NAA was 0.65 .

\section{DISCUSSION}

MNVT was first described as a histopathologically different, purely neuronal benign entity by Huse et al. (8). WHO categorized MVNT under the neuronal and mixed neuronalglial tumors of the 2016 CNS tumor classification. MVNT was considered a low-grade lesion, possibly related to ganglion cell tumors or even a malformation (10). In 2019, the definition was updated, and it was considered a distinctive neuronal tumor (14).

Since the first description of MVNT, two extensive case studies, including 24 and 33 cases, have been published in the radiology literature and some case reports $(1,13)$. However, these reports do not mention the advanced MRI findings of the lesions. In this study, the MR spectroscopy and perfusion characteristics of MVNT are demonstrated in addition to the conventional MRI findings.

MVNT is usually asymptomatic and detected incidentally (1). In symptomatic patients, seizures and seizure equivalents are the most common symptoms. Since the condition is mostly asymptomatic, the real prevalence is not known. Furthermore, being a newly defined entity, the disease can be misdiagnosed because of the relatively low awareness. Lesions are usually supratentorial, located in the temporal lobe. The literature describes a few cases where the lesions are localized in the hippocampus $(8,16)$. One of the cases described here also involved the hippocampus and parahippocampal gyrus.

The lesions are usually not evident in a CT. Yet, as described by Nagaishi et al., and Osborn et al., in some cases, the lesion can be detected as a non-calcified, non-enhancing hypoattenuating lesion in the subcortical white matter $(12,13)$.

MRI manifestations of the lesions are specific and diagnostic. These are typically clumped multinodular lesions with satellite nodules located in the deep cortical ribbon and superficial subcortical white matter. Lesions are unilateral with no mass effect. They are iso-hypointense on $\mathrm{T} 1$, hyperintense on T2 weighted, and FLAIR sequences. The lesions are not tumefactive because of the partial volume effect, even if the small nodules show coalescence. The lesions typically do not show contrast enhancement or diffusion restriction $(9,13)$. However, the literature describes one lesion that exhibited minimal enhancement (13). Susceptibility weighted imaging does not demonstrate intratumoral hemorrhage or calcification $(1,9)$.

The literature reports only a little information about the spectroscopic findings of MNVT. Lecler et al. stated that there is no Cho peak with minimal decrease in NAA; thus, enabling the radiologists to diagnose the lesion (9) confidently. Neither of the two cases described by Gokce revealed a meaningful metabolite peak (7). In one of the cases described in this paper, a prominent rise in Cho's peak with a decrease in NAA (Cho/NAA 2) was observed. Similarly, in the histopathologically confirmed MVNT case presented by Nagashi et al., the MR spectroscopy revealed an increased Cho peak and a decreased NAA. The patient was operated based on suspicion of a glial tumor. Even though the case was not histopathologically confirmed, the MRI findings were typical for MVNT, and no progression in the one-year followup.

In the other three cases, an increased Cho was detected, although not as prominent as the previous case, with decreased NAA. In the case presented by Huse et al. and in the other three cases in the literature, a relative increase in Cho peak and a decrease in NAA was reported $(5,8,11)$. While Huse and Fukushima did not provide a numerical value, Makrakis said a Cho/Cr 1.36 and a Cho/NAA 0.77 in the lesion 
Turan A. et al: Multinodular and Vacuolating Neuronal Tumor

while they were 0.87 and 0.51 consecutively in the normal brain parenchyma. Badat et al. reported a mild decrease in NAA peak (2). Increased Cho makes it difficult for the radiologists to report the lesions as benign MNVT. Still, typical conventional $\mathrm{MRI}$ findings and the absence of progression in the follow-up helps distinguish MNVT.

Although the literature does not report sufficient information concerning MR perfusion, Lecler et al. emphasized that hyperperfusion was not identified in the lesion (9). On the other hand, Gokce and Makrakis reported a slight increase in perfusion $(7,11)$. In another case, slightly decreased CBV values were observed compared to the symmetrical brain parenchyma $(6,7)$. In one of the cases reported in the present paper, a decrease in perfusion was noted compared to the symmetrical brain parenchyma.

The heterogeneous results of the MR spectroscopy and perfusion findings of MNVT demonstrate that generalization of this lesion's advanced MRI findings is challenging at this point and require experience from more such cases.

Histologically, MVNT is located superficially, affecting the deep cortical ribbon and the adjacent white matter. The lesions are in the form of well-defined, multiple, millimetric oval-round nodules, exhibiting coalescence. The endoplasmic reticulum, showing vacuolating degeneration, is common, besides extracellular vacuolation. The cells do not show mitosis or atypia. The area surrounding the brain parenchyma is usually normal but can also be gliotic on rare occasions $(3,8,12)$.

MVNT is positive for some neuronal markers like OLIG2 and synaptophysin but negative for GFAP and NeuN. MVNT is delineated as a neoplasm that causes activation of the RasRaf-MAP kinase signaling pathway. Genetic features of MVNT depict familiarity with gangliogliomas, pilocytic astrocytomas, and dysembryoplastic neuroepithelial tumors (14).

The primary differential diagnosis of MVNT is dysembryoplastic neuroepithelial tumors (DNETs). DNET is commonly localized in the temporal and frontal lobes, affecting the cortex with a bubbly appearance. The bright rim sign and mixed-signal intensity in FLAIR, with some parts being suppressed and contrast enhancement, make the differential diagnosis easier. MR spectroscopy findings are non-specific; a lactate peak can be seen $(6,13)$. Other lesions to be considered in the differential diagnosis are ganglion cell tumors (gangliocytoma/ ganglioglioma) (5), low-grade glioma (6), focal cortical dysplasia (FCD), and periventricular space (PVS) (13). Transmantle signs accompanying FCD may help in the differential diagnosis (13). The typical PVS locations, cystic appearance in T2 weighted sequences, and symmetric distribution are helpful in the diagnosis $(1,6)$. Low-grade gliomas are white matter lesions with mass effect and minimal contrast enhancement (4). Ganglion cell tumors are usually located in the temporal lobe cortex. They contain cystic and solid components, which can show enhancement and can calcify (15).

MVNT can rarely be symptomatic and present with seizures or seizure equivalents. Asymptomatic cases are detected incidentally. MVNT is defined as a "leave me alone" lesion and does not require biopsy or surgery in asymptomatic patients (13).

\section{CONCLUSION}

The clinical characteristics of MNVT are non-specific, but conventional MRI findings are leading and diagnostic. However, advanced MR imaging findings are still not agreed upon, and the radiologists should consider this to provide the correct diagnosis and prevent unnecessary interventional and surgical approaches.

\section{ACKNOWLEDGMENTS}

We thank MRI technician Erdeniz Yurdakul for his valuable contribution to our study and imaging of our patients.

\section{REFERENCES}

1. Alsufayan R, Alcaide-Leon P, de Tilly LN, Mandell DM, Krings T: Natural history of lesions with the MR imaging appearance of multinodular and vacuolating neuronal tumor. Neuroradiology 59:873-883, 2017

2. Badat N, Savatovsky J, Charbonneau F, Collin A, Lecler A: Multinodular vacuolating and neuronal tumor of the cerebrum. Neurology 89:304-305, 2017

3. Bodi I, Curran O, Selway R, Elwes R, Burrone J, Laxton R, Al-Sarraj S, Honavar M: Two cases of multinodular and vacuolating neuronal tumour. Acta Neuropathol Commun 2:7, 2014

4. Forst DA, Nahed BV, Loeffler JS, Batchelor TT: Low-grade gliomas. Oncologist 19:403-413, 2014

5. Fukushima S, Yoshida A, Narita Y, Arita H, Ohno M, Miyakita $\mathrm{Y}$, Ichimura K, Shibui S: Multinodular and vacuolating neuronal tumor of the cerebrum. Brain Tumor Pathol 32:131-136, 2015

6. Gonzalez-Quarante LH, Ruiz-Juretschke F, Sola Vendrell E, Gil de Sagredo Del Corral OL, Agarwal V, Garcia-Leal R: Multinodular and vacuolating neuronal tumor of the cerebrum. A rare entity. New case and review of the literature. Neurocirugia 29:44-55, 2018

7. Gokce E: Magnetic resonance imaging findings of two cases with multinodular and vacuolating neuronal tumor. Acta Neurol Belg 120:457-461, 2020

8. Huse JT, Edgar M, Halliday J, Mikolaenko I, Lavi E, Rosenblum MK: Multinodular and vacuolating neuronal tumors of the cerebrum: 10 cases of a distinctive seizure-associated lesion. Brain Pathol 23:515-524, 2013

9. Lecler A, Bailleux J, Carsin B, Adle-Biassette H, Baloglu S, Bogey C, Bonneville F, Calvier E, Comby PO, Cottier JP, Cotton F, Deschamps R, Diard-Detoeuf C, Ducray F, Duron L, Drissi C, Elmaleh M, Farras J, Garcia JA, Gerardin E, Grand S, Jianu DC, Kremer S, Magne N, Mejdoubi M, Moulignier A, Ollivier M, Nagi S, Rodallec M, Sadik JC, Shor N, Tourdias T, Vandendries C, Broquet V, Savatovsky J; ENIGMA Investigation Group (EuropeaN Interdisciplinary Group for MVNT Analysis): Multinodular and vacuolating posterior fossa lesions of unknown significance. Am J Neuroradiol 40:16891694,2019 
Turan A. et al: Multinodular and Vacuolating Neuronal Tumor

10. Louis DN, Perry A, Reifenberger G, von Deimling A, FigarellaBranger D, Cavenee WK, Ohgaki H, Wiestler OD, Kleihues P, Ellison DW: The 2016 World Health Organization Classification of tumors of the central nervous system: A summary. Acta Neuropathol 131:803-820, 2016

11. Makrakis D, Veneris S, Papadaki E: Multinodular and vacuolating neuronal tumor incidentally discovered in a young man: Conventional and advanced MRI features. Radiol Case Rep 13:960-964, 2018

12. Nagaishi M, Yokoo H, Nobusawa S, Fujii Y, Sugiura $Y$, Suzuki R, Tanaka Y, Suzuki K, Hyodo A: Localized overexpression of alpha-internexin within nodules in multinodular and vacuolating neuronal tumors. Neuropathology 35:561-568, 2015

13. Nunes RH, Hsu CC, da Rocha AJ, do Amaral LLF, Godoy LFS, Watkins TW, Marussi VH, Warmuth-Metz M, Alves HC, Goncalves FG, Kleinschmidt-DeMasters BK, Osborn AG: Multinodular and vacuolating neuronal tumor of the cerebrum: A new "leave me alone" lesion with a characteristic imaging pattern. Am J Neuroradiol 38:1899-1904, 2017
14. Pekmezci M, Stevers M, Phillips JJ, Van Ziffle J, Bastian BC, Tsankova NM, Kleinschmidt-DeMasters BK, Rosenblum MK, Tihan T, Perry A, Solomon DA: Multinodular and vacuolating neuronal tumor of the cerebrum is a clonal neoplasm defined by genetic alterations that activate the MAP kinase signaling pathway. Acta Neuropathol 135:485-488, 2018

15. Shin JH, Lee HK, Khang SK, Kim DW, Jeong AK, Ahn KJ, Choi CG, Suh DC: Neuronal tumors of the central nervous system: Radiologic findings and pathologic correlation. Radiographics 22:1177-1189, 2002

16. Yamaguchi M, Komori T, Nakata $Y$, Yagishita A, Morino $M$, Isozaki E: Multinodular and vacuolating neuronal tumor affecting amygdala and hippocampus: A quasi-tumor? Pathol Int 66:34-41, 2016 